\title{
Diversité des Insectes sur les Panicules de Zea mays (Poaceae) et Leur Impact sur le Rendement Grainier à Maroua (Extrême - Nord, Cameroun)
}

\author{
Joseph Blaise Pando, \\ Denis Djonwangwé, \\ Université de Maroua, Ecole Normale Supérieure, \\ Département des Sciences de la Vie et de la Terre, Cameroun \\ Fernand-Nestor Tchuenguem Fohouo, \\ Université de Ngaoundéré, Faculté des Sciences, \\ Département des Sciences Biologiques, Cameroun \\ Joseph Lebel Tamesse, \\ Université de Yaoundé I, Ecole Normale Supérieure, \\ Département des Sciences Biologiques, Cameroun
}

\section{Doi: 10.19044/esj.2019.v15n9p460 URL:http://dx.doi.org/10.19044/esj.2019.v15n9p460}

\section{Résumé}

A Maroua (Cameroun), 80 panicules de Zea mays L. (Poaceae) ont été observées du 30 août au 16 septembre 2015 et du 5 au 22 Septembre 2016 en vue de déterminer la diversité et la fréquence de visites d'insectes. 10782 visites de 19 espèces d'insectes regroupées dans cinq ordres ont été répertoriées sur les panicules de cette Poaceae. Les Hyménoptères ont été les plus prépondérants représentés principalement par Amegilla sp. avec 94,52 $\%$ de l'ensemble de visites. Les insectes butinaient les panicules du maïs de $6 \mathrm{~h}$ à $11 \mathrm{~h}$ avec le pic de l'activité de l'ensemble de visites des insectes situé entre $6 \mathrm{~h}$ et $7 \mathrm{~h}$ et pendant toute la période de forte floraison. Ces divers insectes développent un comportement très élaboré lors de la récolte du pollen de maïs. Ils ont un impact positif sur les rendements en grains du maïs, qui s'explique par une action complémentaire de celle du vent. Le rôle est indirect puisque ces insectes ne visitent pas les stigmates. Il s'explique par le fait que les insectes très abondants sur les panicules secouent les anthères, provequent ainsi la libération du pollen dans l'air même en l'absence de vent. L'influence des insectes sur l'augmentation des rendements est estimée à 43,16\%, 49,17\% et 76,48\% sur le nombre de grains par épi, la masse des grains par épi et le pourcentage de grains normaux respectivement. Nous recommandons que pour une meilleure gestion intégrée des exploitations de maïs au Cameroun, de préserver les insectes floricoles non nuisibles dans les plantations en évitant les 
traitements aux pesticides pendant la période de floraison lorsqu'ils ne se justifient pas.

Mots-clés: Zea mays, panicules, insectes, pollinisation, rendement, Maroua

\title{
Insect Diversity on the Panicles of Zea mays (Poaceae) and Their Impact on Seed Yield in Maroua (Far-North, Cameroon)
}

\author{
Joseph Blaise Pando, \\ Denis Djonwangwé, \\ Université de Maroua, Ecole Normale Supérieure, \\ Département des Sciences de la Vie et de la Terre, Cameroun \\ Fernand-Nestor Tchuenguem Fohouo, \\ Université de Ngaoundéré, Faculté des Sciences, \\ Département des Sciences Biologiques, Cameroun
}

Joseph Lebel Tamesse,

Université de Yaoundé I, Ecole Normale Supérieure,

Département des Sciences Biologiques, Cameroun

\begin{abstract}
At Maroua (Cameroon), 80 panicles of Zea mays L.(Poaceae) were observed from the $30^{\text {th }}$ August to $16^{\text {th }}$ September 2015 and from $5^{\text {th }}$ to $22^{\text {th }}$ September 2016 to determine the diversity and frequency of insect visits. 10782 visits of 19 insect species grouped in five orders were recorded on the flowers of maize. The Hymenoptera were most dominant particularly Amegilla sp.with $94.52 \%$ of the number of insect visits. The insects explored the panicle of maize during the morning from 6 am to 11 am with the peak activity is observed between 6 am and 7 am during the flowering period of the host plant. These insects developed an elaborated behaviour when they are collected the maize pollen. They have a positive impact on the grain yields of the maize, which can be justified by their complementary action to that of the wind.The action is indirect because the insects do not visit the female inflorescences. This can be explained by the fact that visit of insects on the flowers shooke the anthers, thus inducing the pollen to be released in the air even in the absence of wind.The influence of the insects on the increasingof maize yields is estimated at $43.16 \%, 49.17 \%$ and $76.48 \%$ for the number of seeds per spike, mass of seeds per spike and percentage of
\end{abstract}


normal seeds respectively. We recommend that, for a better integrated management of the maize exploitations into Cameroun, to preserve the flower-dwelling insects in the farm by avoiding pesticide treatments during the period of flowering when they are not justified.

Keywords: Zea mays, panicles, insects, pollination, yield, Maroua

\section{Introduction}

Le maïs constitue la première céréale cultivée dans le monde en termes de quantité et de surface, devant le blé et le riz (Marceau, 2010). Ses grains sont utilisés dans les aliments destinés à la consommation humaine et autres animaux, et comme matière première dans des produits industriels (Hamel \& Dorff, 2014). La production mondiale de grains de maïs est estimée à 885,3 millions de tonnes (FAO, 2014). La production du Cameroun est évaluée à 1647767 tonnes et celle de la région de l'Extrême-Nord est de 144654 (MINADER, 2012). Cette production est insuffisante pour satisfaire la demande de ce pays qui est estimée à 3143116 tonnes (DSCE, 2009). Pour le fonctionnement de ses agro-industries, le Cameroun importe du maïs (FAO, 2014). Au regard de cette situation il est important de chercher dans quelle mesure on peut utiliser les insectes floricoles afin d'augmenter cette production dans ce pays. En effet il est connu que les insectes jouent une rôle important en agronomie (Klein et al., 2007 ; Arem, 2011).

La pollinisation de Z. mays est tributaire de l'action du vent (Mc. Gregor, 1976 ; Pesson et Louveaux, 1984). En effet, le pollen du maïs très fin, lisse, sec et pulvérulent est parfaitement adapté à ce mode de transport et se prête mal à la récolte par les insectes (Vallardir, 1964 ; Zandonella, 1984). Néanmoins des études menées au Burkina-Faso par Pauly (1984), en Thaïlande par Phaya (1985) et en Ethiopie par Fichtl \& Adi (1994) ont montré que les panicules de cette plante sont visitées par Lipotriches rubella et Apis mellifera pour récolter le pollen. Ces auteurs soulignent que ces insectes en contact avec les anthères lors de la récolte du pollen provoquent une vibration de ces derniers entrainant ainsi la libération de milliers de grains de pollens qui participent à la pollinisation de cette plante.

$\mathrm{Au}$ Cameroun, à l'exception des travaux faits par Michener (1969) à Bamenda, Tchuenguem et al. (2002) et Dounia et al. (2018) à Yaoundé, aucune autre production scientifique sur la pollinisation entomophile de $Z$. mays n'est disponible. Ces auteurs montrent que la visite de panicules par les insectes augmente le rendement en grains de cette Poaceae. L'abondance et la diversité des insectes floricoles d'une plante pouvant varier d'une région à une autre (Roubik, 2000), il serait important de mener des investigations supplémentaires à Maroua sur Z. mays pour compléter les données existantes au Cameroun. 
Le présent travail est dont une contribution à la connaissance des relations entre $Z$. mays et les insectes anthophiles en vue de sa gestion optimale au Cameroun. Il s'agit specifiquement: (a) de déterminer la diversité de l'entomofaune floricole de Z. mays; (b) d'évaluer la fréquence de visites de ces insectes sur les panicules du maïs ; (c) d'évaluer l'impact des insectes sur la pollinisation et le rendement en grains de cette Poacée.

\section{Matériel et méthodes}

\section{Site d'étude et matériel biologique}

Les travaux se sont déroulés à Palar $\left(10^{\circ} 36^{\prime} 16.7^{\prime}{ }^{\prime} \mathrm{N}, 14^{\circ} 16^{\prime} 36.5^{\prime}\right.$ 'E et $416 \mathrm{~m})$ en 2015 et à Wourndé $\left(10^{\circ} 38^{\prime} 15.7^{\prime}{ }^{\circ} \mathrm{N}, 1^{\circ} 18^{\prime} 40.4^{\prime \prime} \mathrm{E}\right.$ et $\left.437 \mathrm{~m}\right)$ en 2016, deux quartiers de la ville de Maroua (Extrême - Nord ; Cameroun). Ces coordonnées ont été obtenues à l'aide d'un GPS de marque GARMIN etrex 10. Le matériel végétal est constitué des pieds de $Z$. mays de la variété blanche issus de la germination des graines fournies par l'IRAD de Maroua. Le matériel animal est constitué de l'ensemble des insectes naturellement présents dans l'environnement et qui visitaient les panicules de $Z$. mays.

\section{Méthode}

\section{Préparation, semis et entretien de la parcelle expérimentale}

La parcelle expérimentale était une aire rectangulaire de $21 \mathrm{~m}$ de long sur $13 \mathrm{~m}$ de large. Cette parcelle était subdivisée en quatre blocs de $9 \mathrm{~m}$ de longueur et $5 \mathrm{~m}$ de largeur chacun et séparés les uns des autres par des allées de $1 \mathrm{~m}$. Le 13 juillet 2015 et le 15 juillet 2016, les opérations suivantes ont été successivement effectuées sur les parcelles expérimentales : défrichement, labour à la charrue et formation des blocs. Le 15 juillet 2015 et 17 juillet 2016, les semis ont été faits, en ligne sur les blocs, à raison de 10 lignes par bloc. Les graines étaient semées à raison de trois par poquet, l'espacement était de $80 \mathrm{~cm}$ entre les poquets et $50 \mathrm{~cm}$ entre les lignes et à 4 $\mathrm{cm}$ de profondeur. Le démariage a été fait lorsque les plantes portaient quatre feuilles et seules deux plantes du maïs ont été conservées par poquet. De la germination survenue (19 juillet 2015 et 21 juillet 2016) à l'apparition des premières inflorescences (30 août 2015 et 3 septembre 2016), les opérations de sarclage à la houe ont été effectuées régulièrement toutes les deux semaines. De la période de floraison jusqu'à la maturation des épis (30 août au 26 septembre 2015 et du 5 septembre au 2 octobre 2016), le désherbage était fait régulièrement à la main. 


\section{Détermination de la diversité et de la fréquence des insectes sur la panicule de Zea mays}

Le 29 août 2015 et le 3 septembre 2016, 320 plants au stade de préfloraison ont été étiquetés et quatre lots constitués : lots 1(2015) et 1' (2016), constitués chacun de 40 plants non protégés, c'est-à-dire dont les panicules et les épis sont libres (Figure 1a) pour la mise en évidence de la pollinisation mixte (autopollinisation et pollinisation croisée) ; lots 2 (2015) et 2'(2016), constitués chacun de 40 plants protégés délicatement à l'aide des sacs en toile gaze (Figure 1b) pour la mise en évidence de l'autopollinisation ; lots 3 (2015) et 3' (2016), constitués chacun de 40 plants dont les panicules ont été supprimées et les épis ont été protégés à l'aide des papiers kraft (Figure 1c) pour la mise en évidence de la parthénocarpie; lots 4 (2015) et 4' (2016), constitués chacun de 40 plants dont les panicules ont étés supprimées et les épis (soies) laissés en libre pollinisation (Figure 1d) pour la mise en évidence de la pollinisation croisée.

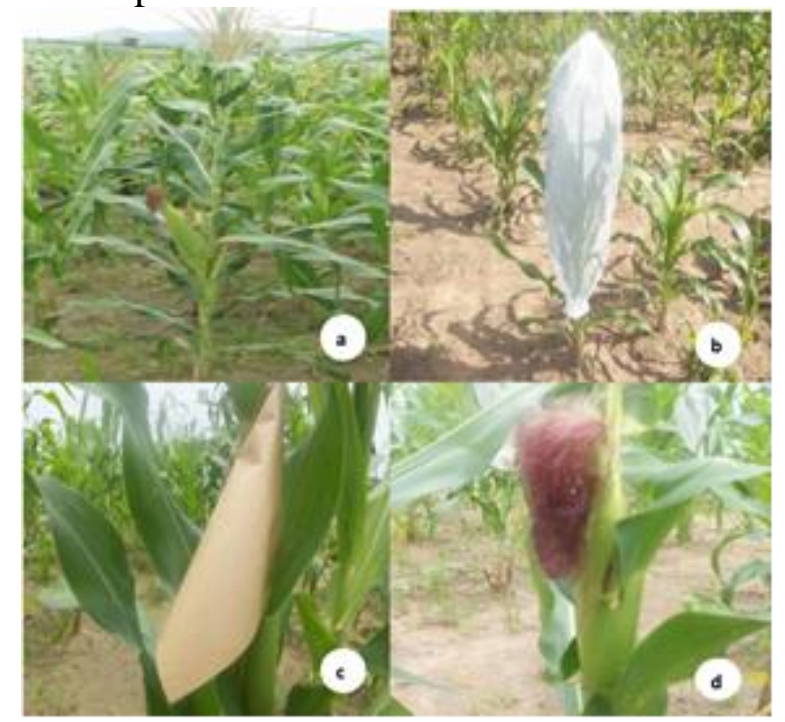

Figure 1 : Différents traitements appliqués sur Zea mays $(\mathbf{a}:$ plante à panicule et à épi non protégés ; $\mathbf{b}$ : pied protégé à l'aide de sac en toile gaze ; $\mathbf{c}:$ pied dont la panicule supprimée et épi protégé à l'aide du papier kraft ; $\mathbf{d}$ : pied dont la panicule supprimée et l'épi laissé en libre pollinisation).

Pour déterminer la diversité des insectes, durant la période d'étude, les insectes ont été capturés sur les panicules non protégées et ceci à la main et/ou au filet entomologique. Sur le terrain, les insectes capturés ont été conservés dans des flacons contenant de l'éthanol à $70 \%$. Sur chaque spécimen, il a été noté l'heure, la date et le lieu de capture. Pour les espèces non identifiées avec précision sur le terrain, il leur a été attribué un code et une description pour faciliter les observations. La détermination des spécimens a été faite au Laboratoire de Sciences Biologiques de Ecole 
Normale Supérieure de l'Université de Maroua à l'aide des clés d'identification (Delvare et Arbelenc, 1989 ; Eardley et al., 2010 ; Pauly, 2014).

Pour déterminer la fréquence de visites de chaque insecte, les observations ont été effectuées sur les panicules de chacun des plants des lots 1 et 1', tous les jours du 30 août au 16 septembre 2015 et du 5 au 22 septembre 2016 respectivement, pendant trois périodes journalières : $6 \mathrm{~h}-7 \mathrm{~h}$, 8h-9h, 10h-11h. Au cours de chaque journée d'investigation, l'observateur passait une fois sur chaque panicule du lot non protégé, pour chacune des périodes journalières ci-dessus. A chaque passage, les différents insectes étaient comptés sur les panicules épanouies (c'est-à-dire celles pouvant offrir $\mathrm{du}$ pollen aux insectes). Les insectes n'étant pas marqués, les résultats cumulés sont exprimés par le nombre de visites (Tchuenguem et al., 2001). Les données obtenues ont permis de déterminer la fréquence de chaque espèce d'insecte $(F i)$ sur les panicules de $Z$. mays selon la formule ci-après : $F i=\{[(V i) / V I] * 100\}(1)$, avec $V i$ le nombre de visite de l'insecte i sur les panicules du lot libre et $V I$ le nombre de visites de tous les insectes sur ces mêmes panicules (Tchuenguem et al., 2001).

\section{Détermination de l'impact des insectes sur la pollinisation de Zea mays}

L'évaluation de l'impact des insectes sur la pollinisation de Z. mays a été faite tout au long de l'étude de la fréquence des visites. Il s'agissait de noter le nombre de fois où le corps de l'insecte entre en contact avec les anthères de la panicule visitée et que le pollen soit expulsé est tombé sur les stigmates. C'est ce qui a permis de mettre en évidence la participation des insectes dans l'autopollinisation et la pollinisation croisée (Tchuenguem et $a l ., 2001)$. Pour déterminer les différents catégories de pollinisateurs, l'indice de régularité $(\mathrm{R})$ a été calculé à l'aide la formule : $R=[(P / 100)$ * $(f / 100)]$ (Tchuenguem, 1993) (2), où $P$ et $f$ sont le pourcentage des visites de l'insecte et la fréquence relative des visites de l'insecte.

\section{Détermination de l'impact des insectes sur les rendements de Zea mays}

L'évaluation de l'impact des insectes pollinisateurs sur les rendements de Z. mays est basée sur la comparaison des rendements grainiers des lots 1 (panicules et épis non protégés) et 2 (panicules protégées des visites d'insectes). A la maturité, le nombre moyen de grains par épi, la masse moyenne des grains par épi et le nombre de grains normaux ont été déterminés.

Le pourcentage $(P g)$ du nombre de grains par épi attribuable aux insectes floricoles est calculé à l'aide de la formule : $P g=\{[(g 1-g 2) / g 1]$ * $100\}$ (3) où $g 1$ et $g 2$ sont les nombres moyens de grains par épi dans les lots 1 et 2 respectivement. 
Le pourcentage $(\mathrm{Pm})$ de masse moyenne des grains par épi attribuable aux insectes floricoles est calculé à l'aide de la formule :Pm $=\{[(m 1-m 2) /$ $m l] * 100\}$ (4) où $m l$ et $m 2$ sont les masses moyennes des grains par épi issus des lots 1 et 2 respectivement.

Le pourcentage $(P n)$ de grains normaux dû à l'influence des insectes floricoles est calculé à l'aide de la formule : $P n=\{[(g n l-g n 2) / g n 1] * 100\}$ (5) où gnl et gn2 sont les pourcentages de grains normaux issus des lots 1 et 2 respectivement.

\section{Traitement des données}

Le traitement des données a été fait à l'aide de la statistique descriptive: (calcul des moyennes, écart- types, pourcentages), du test $\mathrm{Z}$ pour la comparaison de deux moyennes, de l'ANOVA pour la comparaison de plus de deux moyennes, du Khi-carré $\left(\chi^{2}\right)$ pour la comparaison des pourcentages (Schwartz, 1984), et du logiciel SPSS 19.0.

\section{Résultats et discussion}

\section{Diversité et fréquence de l'entomofaune floricole de Zea mays}

Au cours des périodes de floraison en 2015 et en 2016, 5339 visites de 10 espèces d'insectes et 5443 visites de 16 espèces d'insectes ont été recensées sur les panicules de 40 pieds et 40 pieds de maïs en 18 jours et 18 jours respectivement (Tableau 1). Dans l'ensemble, 19 espèces insectes ont été inventoriées durant les deux investigations. Cette diversité spécifique est inférieure à celle obtenue à Yaoundé par Tchuenguem et al. (2002) qui était de 35 espèces d'insectes. Ceci pourrait s'expliquer par la différence écologique existant entre les deux zones d'études. En effet, Maroua est dans la zone sahélienne et Yaoundé dans la zone équatoriale. Ceci confirme les travaux de Roubik (2000) qui stipule que la diversité de l'entomofaune floricole d'une plante varie dans le temps et dans l'espace.

Tableau 1 : Insectes recensés sur les panicules de Zea mays en 2015 et en 2016 et leur fréquence de visites en 36 jours d'observation à Maroua

\begin{tabular}{|c|c|c|c|c|c|c|c|c|}
\hline \multicolumn{2}{|l|}{ Insectes } & & \multicolumn{2}{|c|}{ Année 2015} & \multicolumn{2}{|c|}{ Année 2016} & \multicolumn{2}{|l|}{ Total } \\
\hline Ordres & Familles & Genres et espèces & $n_{1}$ & $P_{1}$ & $n_{2}$ & $P_{2}$ & $n_{t}$ & $P_{t}$ \\
\hline \multirow[t]{12}{*}{ Hyménoptères } & Apidae & Amegilla sp. & 5088 & 95,30 & 5103 & 93,75 & 10191 & 94,52 \\
\hline & Halictidae & Sphecodes albilabris & 37 & 00,69 & 54 & 00,99 & 91 & 00,84 \\
\hline & & Lipotriches collaris & 122 & 02,29 & 109 & 02,00 & 231 & 02,14 \\
\hline & & Lasioglossum albipes & 65 & 01,22 & 78 & 01,43 & 143 & 01,33 \\
\hline & & Lasioglossum sp. & - & - & 17 & 00,31 & 17 & 00,16 \\
\hline & & Seladonia tripartitus & - & - & 21 & 00,39 & 21 & 00,19 \\
\hline & Sphecidae & Larra sp. & - & - & 09 & 00,17 & 09 & 00,08 \\
\hline & & Prionyx atratus & - & - & 03 & 00,06 & 03 & 00,03 \\
\hline & Vespidae & Trichinothorax sp. & 06 & 00,11 & 09 & 00,16 & 15 & 00,14 \\
\hline & & (Eumeninae) 1sp. & 05 & 00,09 & - & - & 05 & 00,05 \\
\hline & & $\begin{array}{l}\text { Belonogaster juncea } \\
\text { juncea }\end{array}$ & - & - & 05 & 00,09 & 05 & 00,05 \\
\hline & Fourmicidae & Camponotus & 05 & 00,09 & 11 & 00,20 & 16 & 00,15 \\
\hline
\end{tabular}




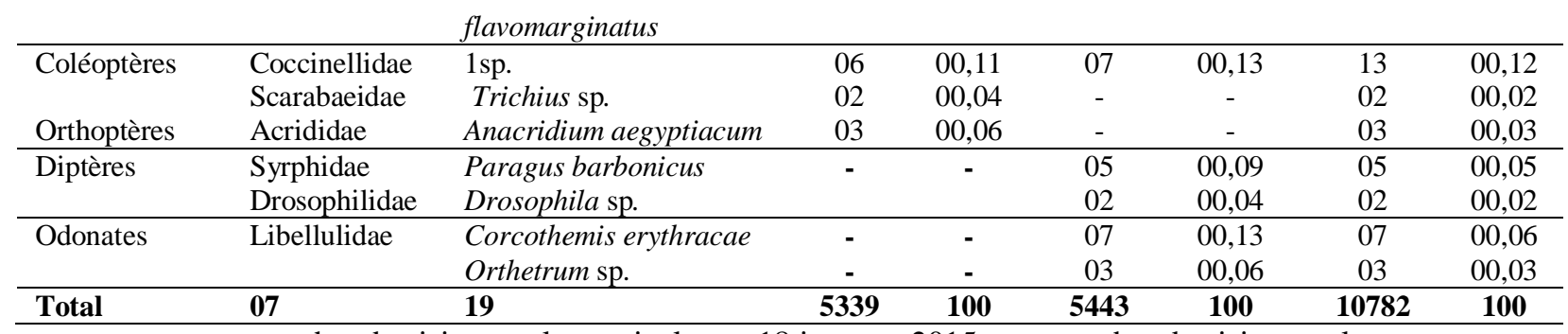

$n_{1}:$ nombre de visites sur les panicules en 18 jours en $2015 ; n_{2}:$ nombre de visites sur les panicules en 18 jours en $2016 ; n_{t}$ : nombre de visites sur les panicules en 36 jours en 2015 et en $2016 ; P_{1}$ : pourcentage de visite en $2015, P_{2}$ : pourcentage de visite en $2016 ; P_{2}$ : pourcentage de visite en 2015 et en 2016 avec $P_{l}=\left(n_{1} / 5339\right) * 100 ; P_{2}=\left(n_{2} / 5443\right) * 100$;

$$
P_{t}=\left(n_{t} / 10782\right) * 100
$$

Il ressort de ce tableau que cinq ordres d'insectes visitent les panicules de maïs: Hyménoptères, Coléoptères, Orthoptères, Diptères et Odonates. L'ordre des Hyménoptères est le plus prépondérant avec 99,82\% des visites. Ils sont surtout représentés par les Apidae notamment Amegilla sp. avec 97,17 \% (Figure 2), suivi des Halictidae parmi lesquels il y a Lipotriches collaris avec 01,17 \%. Les autres insectes sont très peu rencontrés. Ces résultats sont similaires aux travaux de Tchuenguem (1993) à Yaoundé qui a signalé que les Hyménoptères étaient les visiteurs les plus communs des panicules de Z. mays avec 95,20\% des visites d'insectes représentés notamment par les Apidae et les Halictidae.

Du tableau 1, il ressort également que parmi les insectes recensés, Drosophila sp., Trichius sp., Orthetrum sp., Prionyx atratus, et Paragus barbonicus ont les plus faibles pourcentages des visites $(0,02 \%)$. Le pourcentage élevé des visites de Amegilla sp. pourrait être lié à la grande dépendance de cet insecte vis-à-vis du pollen de Z. mays. Par contre, Phaya (1985) en Thaillande, Fichtl etAdi (1994) en Ethiopie ont signalé que c'est l'Abeille Apis mellifera qui était l'insecte butineur le plus actif sur les panicules de cette plante dans ces pays. 


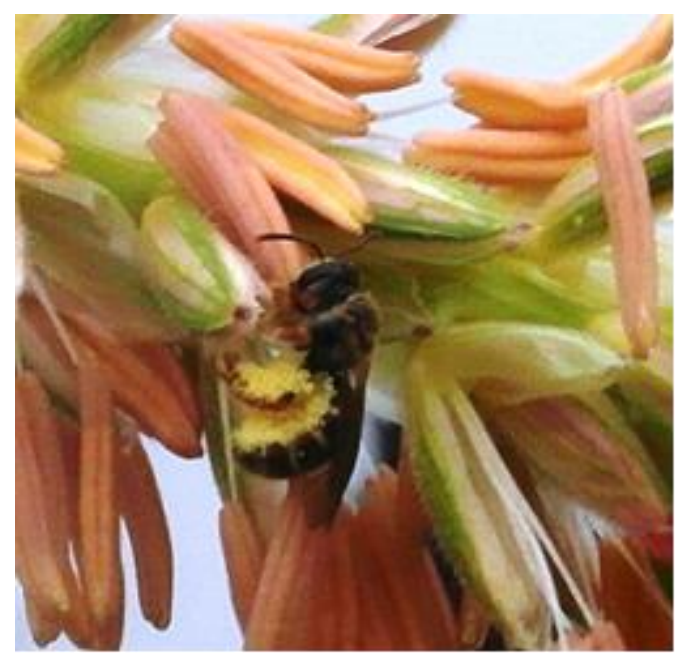

Figure 2 : Amegilla sp. récoltant du pollen sur une panicule de Zea mays

Dans l'ensemble, pendant 36 jours d'observation, les insectes sont rencontrés quotidiennement sur les panicules de Z. mays comme l'illustre le tableau 2.

Tableau 2 : Présence, en nombre et en pourcentage des jours, des différents insectes visitant les panicules de Zea mays en 2015 et en 2016 à Maroua

\begin{tabular}{|c|c|c|c|c|c|c|}
\hline \multirow[t]{2}{*}{ Insectes } & \multicolumn{2}{|c|}{ Année 2015} & \multicolumn{2}{|c|}{ Année 2016} & \multicolumn{2}{|c|}{ Total } \\
\hline & $n_{1}$ & $f_{1}(\%)$ & $n_{2}$ & $f_{2}(\%)$ & $f_{t}$ & $f_{t}(\%)$ \\
\hline Amegilla sp. & 18 & 100 & 18 & 100 & 36 & 100 \\
\hline Sphecodes albilabris & 12 & 66,67 & 09 & 50,00 & 21 & 58,33 \\
\hline Lipotriches collaris & 11 & 61,11 & 16 & 88,89 & 27 & 75,00 \\
\hline Lasioglossum albipes & 07 & 38,89 & 11 & 61,11 & 18 & 50,00 \\
\hline Lasioglossum sp. & - & - & 07 & 38,89 & 7 & 19,44 \\
\hline Seladonia tripartitus & - & - & 11 & 61,11 & 11 & 30,56 \\
\hline Larra sp. & - & - & 03 & 16,67 & 3 & 08,33 \\
\hline Prionyx atratus & - & - & 01 & 05,56 & 1 & 02,78 \\
\hline Trichinothorax sp. & 02 & 11,11 & 04 & 22,22 & 6 & 16,67 \\
\hline (Eumeninae) sp. & 04 & 22,22 & - & - & 2 & 05,56 \\
\hline Belonogaster juncea juncea & - & - & 03 & 16,67 & 3 & 08,33 \\
\hline Camponotus flavomarginatus & 03 & 16,67 & 04 & 22,22 & 7 & 19,44 \\
\hline (Coccinellidae) sp. & 02 & 11,11 & 02 & 11,11 & 4 & 11,11 \\
\hline Anacridium aegyptiacum & 01 & 05,56 & - & - & 1 & 02,78 \\
\hline Trichius sp. & 02 & 11,11 & - & - & 2 & 05,56 \\
\hline Paragus barbonicus & - & - & 02 & 11,11 & 2 & 05,56 \\
\hline Drosophila $\mathrm{sp}$. & - & - & 01 & 05,56 & 1 & 02,78 \\
\hline Corcothemis erythracae & - & - & 02 & 11,11 & 2 & 05,56 \\
\hline Orthetrum $\mathrm{sp.}$ & - & - & 01 & 05,56 & 1 & 02,78 \\
\hline
\end{tabular}

$n_{1}$ : Nombre de jours de présence d'insectes pendant $\mathrm{N}_{1}$ jours d'observation en $2015 ; n_{2}$ :

Nombre de jours de présence d'insectes pendant $\mathrm{N}_{1}$ jours d'observation en $2016 ; n_{2}$ :

Nombre de jours de présence d'insectes pendant $\mathrm{N}_{\mathrm{t}}$ jours d'observation en 2015 et $2016 ; f_{l}$

$(\%)$ : Fréquence relative des visites de l'insecte $\left(n_{1} / N_{l}\right)^{*} 100 ; f_{l}(\%)$ : Fréquence relative des visites de l'insecte $\left(n_{2} / N_{2}\right) * 100 ; f_{l}(\%)$ : Fréquence relative des visites de l'insecte $\left(n_{2} /\right.$ $\left.N_{2}\right)^{*} 100 ; N_{l}=N_{2}=18, N_{t}=36$ 
Il ressort de ce tableau que Z. mays a des visiteurs plus ou moins fréquents. Ainsi, il y a: (a) les visiteurs très fréquents avec une fréquence des visites supérieure à $50 \%$ du nombre total des jours d'observations (Tchuenguem, 1993): Amegilla sp., Lipotriches collaris et Sphecodes albilabris; (b) les visiteurs fréquents avec une fréquence des visites comprise entre 25 et $50 \%$ du nombre total des jours d'observations (Tchuenguem, 1993): Lasioglossum albipes et Seladonia tripartitus; (c) les visiteurs rares qui ont une fréquence de visites inférieure à $25 \%$ du nombre total des jours d'observation (Tchuenguem, 1993) qui sont représentés par les autres insectes. Ces résultats nous permettent de supposer que les panicules de $Z$. mays produisent importants grains de pollens ; ce qui fait que les insectes qui y sont rencontrés pendant sa période de floraison lui soient fidèles. Ces stimuli produits par les panicules de cette plante agiraient beaucoup plus sur les insectes pollinisateurs que sur les visiteurs occasionnels des panicules. Des observations similaires ont été faites par Dounia et al. (2018) à Yaoundé.

Il ressort également de ce tableau 2 que la fréquence de présence de Amegilla sp. en 36 jours d'observation est de $100 \%$. Ceci dénote du son bon attachement aux pollens de Z. mays. Par contre les travaux de Tchuenguem et al. (2002) et Dounia et al. (2018) à Yaoundé ont trouvé plutôt que c'est Lipotriches andrei et Apis mellifera respectivement qui étaient les abeilles les plus fréquentes sur les panicules $(100 \% \mathrm{du}$ nombre total des jours d'observations) du maïs. Ceci peut être dû à l'absence ou à la faible présence de Amegilla dans cette localité.

\section{Rythme de visites d'insectes selon les tranches horaires journalières}

Les insectes visitaient les panicules de $Z$. mays de 6 heures à 11 heures comme l'indique le tableau 3 à Maroua. Ce résultat est en accord à celui de Pauly (1984) au Burkina Fasso. Il est contraire à ceux de Tchuenguem et al. (2002) à Yaoundé qui ont trouvé plutôt que les insectes sont actifs sur les panicules de cette Poaceae pendant toute la journée. Ceci serait dû aux faibles taux d'humidités relatives qui caractérise dans les après-midis en zone sahélienne qui ne permettent pas la collecte du pollen très pulvérulent de cette espèce végétale.

Tableau 3 : Variation du nombre de visites des insectes sur la panicule de Zea mays par tranche horaire en 36 jours d'observation en 2015 et en 2016 à Maroua.

\begin{tabular}{|c|c|c|c|c|c|c|c|}
\hline \multirow[t]{3}{*}{ Insectes } & \multicolumn{7}{|c|}{ Nombres de visites par tranche horaire } \\
\hline & \multicolumn{2}{|c|}{ 6h-7h } & \multicolumn{2}{|c|}{ 8h-9h } & \multicolumn{2}{|c|}{ 10h-11h } & \multirow[b]{2}{*}{ Total } \\
\hline & $N V_{1}$ & $P_{1}(\%)$ & $N V_{2}$ & $P_{2}(\%)$ & $N V_{3}$ & $P(\%)$ & \\
\hline Amegilla sp. & 9888 & $97,03^{\alpha}$ & 289 & 02,84 & 14 & 00,13 & 10191 \\
\hline Sphecodes albilabris & 28 & 30,77 & 48 & $52,74^{\alpha}$ & 15 & 16,49 & 91 \\
\hline Lipotriches collaris & 197 & $85,28^{\alpha}$ & 28 & 12,12 & 6 & 02,60 & 231 \\
\hline Lasioglossum albipes & 107 & $74,83^{\alpha}$ & 29 & 20,28 & 07 & 04,89 & 143 \\
\hline
\end{tabular}




\begin{tabular}{lccccccc}
\hline Lasioglossum sp. & 11 & $64,71^{\alpha}$ & 05 & 29,41 & 1 & 05,88 & 17 \\
Seladonia tripartitus & 13 & $61,90^{\alpha}$ & 07 & 33,33 & 1 & 04,77 & 21 \\
Larra sp. & 09 & $100^{\alpha}$ & - & - & - & - & 09 \\
Prionyx atratus & 03 & $66,67^{\alpha}$ & 1 & 33,33 & - & - & 03 \\
Trichinothorax sp. & 15 & $100^{\alpha}$ & - & - & - & - & 15 \\
(Eumeninae) sp. & 05 & $100^{\alpha}$ & - & - & - & - & 05 \\
Belonogaster juncea juncea & 04 & $80,00^{\alpha}$ & 1 & 20,00 & - & - & 05 \\
Camponotus flavomarginatus & 6 & 37,50 & 10 & $62,50^{\alpha}$ & - & - & 16 \\
Coccinellidae) sp. & 4 & 30,77 & 7 & $53,84^{\alpha}$ & 2 & 15,39 & 13 \\
Anacridium aegyptiacum & - & - & 03 & $100^{\alpha}$ & - & - & 03 \\
Trichius sp. & 02 & $100^{\alpha}$ & - & - & - & - & 02 \\
Paragus barbonicus & 4 & $80,00^{\alpha}$ & 01 & 20,00 & - & - & 05 \\
Drosophila sp. & 02 & $100^{\alpha}$ & - & - & - & - & 02 \\
Corcothemis erythracae & 5 & $71,42^{\alpha}$ & 1 & 14,29 & 1 & 14,29 & 07 \\
Orthetrum sp. & 1 & 33,33 & 2 & $66,67^{\alpha}$ & - & - & 3 \\
\hline Total & 10304 & $95,57^{\alpha}$ & 431 & 09,99 & 47 & 00,44 & 10782 \\
\hline
\end{tabular}

$N V_{1}$ : nombre de visites entre $6 \mathrm{~h}$ et $7 \mathrm{~h} ; N V_{2}:$ nombre de visites entre $8 \mathrm{~h}$ et $9 \mathrm{~h}$;

$N V_{3}:$ nombre de visites entre $10 \mathrm{~h}$ et $11 \mathrm{~h} ; P_{1}(\%)$ : pourcentage des visites entre $6 \mathrm{~h}$ et $7 \mathrm{~h} ; P_{2}(\%)$ : pourcentage des visites entre $8 \mathrm{~h}$ et $9 \mathrm{~h} ; P_{3}(\%)$ : pourcentage des visites entre $10 \mathrm{~h}$ et $11 \mathrm{~h} ;\left(^{\boldsymbol{\alpha}}\right)$ : pic d'activité de l'insecte

Il ressort également de ce tableau que la période journalière de butinage varie avec les insectes. Les visites de Amegilla sp., Lipotriches collaris, Lasioglossum albipes, Lasioglossum sp., Seladonia tripartitus, Corcothemis erythracae s'étendent de $6 \mathrm{~h}$ à $11 \mathrm{~h}$ avec un pic d'activité situé entre $6 \mathrm{~h}$ et $7 \mathrm{~h}$. Les visites de Prionyx atratus, Belonogaster juncea juncea et Paragus barbonicus s'étendent de $6 \mathrm{~h}$ à $9 \mathrm{~h}$ avec leur pic d'activité situé entre $6 \mathrm{~h}$ et $7 \mathrm{~h}$. Sphecodes albilabris et le Coccinellidae 1sp. sont présent sur les panicules de $6 \mathrm{~h}$ à $11 \mathrm{~h}$, avec le pic d'activité situé entre 8 et $9 \mathrm{~h}$. Trichinothorax sp., Larra sp., Drosophila sp., Trichius sp. et le Eumeninae 1 sp. sont actifs sur les panicules de $Z$. mays uniquement entre $6 \mathrm{~h}$ et 7h. Orthetrum sp., Anacridium aegyptiacum et Camponotus flavomarginatus visitent les panicules de cette plantes de $6 \mathrm{~h}$ à $9 \mathrm{~h}$, avec un pic d'activité situé entre $8 \mathrm{~h}$ et $9 \mathrm{~h}$.

Du tableau 3, il ressort en plus que la période d'activité intense de l'ensemble des insectes se situe entre $6 \mathrm{~h}-7 \mathrm{~h}$, avec 95,57\% de visites. Ceci serait lié d'une part à la forte disponibilité du pollen de cette plante dans la matinée et d'autre part à l'humidité matinale qui sont favorables à l'accumulation du pollen dans les organes du transport de plusieurs insectes. En effet, au fur et à mesure que la température augmente et que l'hygrométrie baisse, le vent peut influencer la disponibilité du pollen sur les panicules de Z. mays. Les grains de pollen du maïs étant pulvérulents, ils sont facilement emportés par le vent au cours de la journée, rendant ainsi les panicules pauvres en pollen. C'est ce qui pourrait expliquer la diminution du 
nombre de visites d'insectes au cours de la journée. Pour Amegilla sp., Lipotriches collaris et Lasioglossum albipes, ces résultats confirment celui de Taséi (1984) qui signalait que l'heure de butinage des Apoïdes solitaires dépend également des disponibilités du pollen à récolter. Le décalage des pics de visites de certains Hyménoptères tels que Sphecodes albilabris et Camponotus flavomarginatus serait une adaptation permettant à chacun d'eux de limiter l'effet de compétition interspécifique lors de la recherche du pollen de Z. mays. La période d'activité optimale de Trichinothorax sp. serait une adaptation permettant à ce prédateur d'accéder à sa proie Amegilla sp. d'où la coüncidence des pics de visites de ces Hyménoptères. En effet, cet insecte cherchait à capturer Amegilla sp. sur les panicules de Z. mays.

\section{Impact des insectes sur la pollinisation de $Z$. mays}

Le nombre et les pourcentages de visites de quelques insectes ayant été en contact permanent avec les anthères de Z. mays durant les 36 jours d'observation sont indiqués dans le tableau 4. Il ressort de ce tableau que tous les insectes ayant des contacts avec les anthères sont des Hyménoptères. Trois de ces insectes ont une fréquence de contacts avec les anthères de $100 \%$. Au cours des visites de panicules, ces insectes secouent celles-ci et favorisent l'ouverture des anthères, provoquant la libération du pollen dans l'air. Une fraction du pollen libéré dans l'air par l'action des insectes est transportée essentiellement par le vent et la gravité jusqu'aux stigmates comme l'avait déjà noté Tchuenguem (1993) à Yaoundé. Par conséquent, ces hyménoptères jouent un rôle positif et indirect sur la pollinisation comme l'avait signalé Phaya (1985). Ceci se comprend aisément puisque l'autogamie et l'allogamie existent chez le maïs. Ces résultats sont en accord avec ceux obtenus par Tchuenguem et al. (2002) qui ont signalés que l'activité de certains Apoïdes sur la panicule de Z. mays favorisait l'autogamie et l'allogamie de cette plante. De plus, l'activité de ces insectes est plus intense dans la matinée, période de déhiscence optimale des anthères et de réceptivité des stigmates du maïs (Tchuenguem et al., 2002). Ces faits favorisent une pollinisation efficace pouvant conduire à une fécondation optimale gage d'un bon rendement en grains. Chaque insecte ayant visité la panicule de $Z$. mays a potentiellement eu un impact positif sur la pollinisation de cette plante. L'indice de régularité (tableau 4) et le comportement spécifique de chaque insecte sur la panicule ont permis de définir les insectes pollinisateurs de cette Poaceae. Ainsi, les différents insectes recensés sur les panicules de Z. mays sont définis en trois catégories de pollinisateurs par Tchuenguem (1993) : 
- pollinisateurs majeurs caractérisés par l'indice de régularité $(R)$ élevé $(R>0,05)$; doublé d'une bonne récolte du pollen : Amegilla sp. $(R=$ $0,95)$ est le seul insecte qui remplis ces conditions..

Tableau 4 : Indice de régularité, nombres et pourcentages de visites des insectes en contact permanent avec les anthères de panicules de Zea mays

\begin{tabular}{|c|c|c|c|c|c|c|}
\hline \multirow[t]{2}{*}{ Insectes } & \multirow{2}{*}{$\begin{array}{c}2015 \\
R_{1} \\
\end{array}$} & \multirow{2}{*}{$\begin{array}{c}2016 \\
R_{2} \\
\end{array}$} & \multirow{2}{*}{$\begin{array}{c}\text { Total } \\
R_{T} \\
\end{array}$} & \multicolumn{3}{|c|}{ NPVCA } \\
\hline & & & & $N V E$ & $N$ & $P(\%)$ \\
\hline Amegilla sp. & 0,9530 & 0,93750 & 0,97000 & 19101 & 19101 & 100 \\
\hline Sphecodes albilabris & 0,0046 & 0,00495 & 0,00270 & 91 & 88 & 96,70 \\
\hline Lipotriches collaris & 0,0139 & 0,01778 & 0,00876 & 231 & 228 & 98,70 \\
\hline Lasioglossum albipes & 0,0466 & 0,00873 & 0,00360 & 143 & 143 & 100 \\
\hline Lasioglossum sp. & - & 0,00121 & 0,00015 & 17 & 13 & 76,47 \\
\hline Seladonia tripartitus & - & 0,00238 & 0,00034 & 21 & 20 & 95,23 \\
\hline Larra sp. & - & 0,00280 & 0,00003 & 09 & 2 & 22,22 \\
\hline Prionyx atratus & - & 0,00003 & 0,00000 & 03 & 0 & 0 \\
\hline Trichinothorax sp. & 0,00011 & 0,00355 & 0,00012 & 15 & 2 & 13,33 \\
\hline (Eumeninae) sp. & 0,00022 & - & 0,00002 & 05 & 0 & 0 \\
\hline Belonogaster juncea juncea & - & 0,00015 & 0,00003 & 05 & 0 & 0 \\
\hline Camponotus flavomarginatus & 0,0001 & 0,00044 & 0,00013 & 16 & 16 & 100 \\
\hline (Coccinellidae) sp. & 0,0001 & 0,00014 & 0,00010 & 13 & 5 & 38,46 \\
\hline Anacridium aegyptiacum & 0,00003 & - & 0,00001 & 03 & 0 & 0 \\
\hline Trichius sp. & 0,00004 & - & 0,00001 & 02 & 0 & 0 \\
\hline Paragus barbonicus & - & 0,00009 & 0,00002 & 05 & 0 & 0 \\
\hline Drosophila sp. & - & 0,00002 & 0,00000 & 02 & 0 & 0 \\
\hline Corcothemis erythracae & - & 0,00014 & 0,00002 & 07 & 2 & 28,57 \\
\hline Orthetrum sp. & - & 0,00003 & 0,00001 & 03 & 2 & 66,67 \\
\hline
\end{tabular}

$R=(P / 100) *(f / 100) ; P$ : pourcentage des visites de l'insecte (tableau I) $; f$ : Fréquence relative des visites de l'insecte $(N / 36) * 100 ; N V E$ : nombre de visites étudiées ; NPVCA : nombre et pourcentage de visites avec contact des anthères, $N^{\prime}:$ nombre de visites avec contact ; $P(\%)$ : pourcentage de visites avec contact.

- pollinisateurs mineurs caractérisés par un indice de régularité faible $(0,05 \leq R<0,001)$ auquel s'ajoute la récolte du pollen: Sphecodes albilabris, Lasioglossum albipes et Lipotriches collaris.

- pollinisateurs occasionnels qui se caractérisés soit par l'indice de régularité très faible $(R<0,001)$ doublé d'une absence de comportement lié à la recherche du pollen, soit par leur capacité à détruire les organes de la panicule. $\mathrm{Ce}$ sont Seladonia tripartitus, Trichinothorax sp, Lasioglossum sp., Larra sp., Prionyx atratus, Trichinothorax sp., Belonogaster juncea juncea, Camponotus flavomarginatus, le Eumeninae 1sp., Anacridium aegyptiacum, Trichius sp., le Coccinellidae 1sp. Paragus barbonicus, Drosophila sp., et Corcothemis erythracae. Environ 19 espèces d'insectes visitent 
les panicules de Z. mays et jouent un rôle certains dans la pollinisation de la Poaceae. Parmi ces insectes, Amegilla sp. joue le rôle prépondérant. Ce résultat est différent de ceux de Tchuenguem (1993) et Dounia et al. (2018) à Yaoundé qui ont signalé que c'est plutôt l'abeille domestique Apis mellifera qui jouait ce rôle. Ceci serait dû à l'absence de la colonie de Apis mellifera dans notre site d'étude. En effet, d'après les travaux de Roubik (2000), les insectes pollinisateurs d'une plante donnée varient d'une région à l'autre.

\section{Impact des insectes sur le rendement grainier de Zea mays}

Le tableau 5 présente le nombre de grains par épi, la masse moyenne des grains par épi et le pourcentage de grains normaux obtenus dans les différents lots.

* Les différences entre les nombres moyens des grains par épi issus des quatre lots en $2015(F 2015=57,85 ; d d l=3 ; P<0,001)$ et en 2016 $(F 2016=57,85 ; d d l=3 ; P<0,001)$ se sont révélées hautement significatives. Les nombres moyens des grains par épi montre que les différences observées sont très hautement significatives entre les lots 1 et $2(Z 2015=5,03 ; d d l=88 ; P<0,001)$ et les lots 1 , et ${ }^{\prime} '(Z 2016=7,23 ; d d l=88 ; P<0,001)$. Le pourcentage $d u$ nombre moyen de grains par épi attribuable à l'influence des insectes floricoles est de 43,16\%. Ce chiffre est supérieur à celui obtenu par Tchuenguem et al. (2002) à Yaoundé sur la même plante qui était de 16,42\%. En analysant le tableau 5, il apparait que le nombre moyen de grains par épi dans les lots 4 et 4' est supérieur au nombre moyen de grains par épi trouvés dans les lots 2 et 2' respectivement. Ceci s'explique par le fait que la pollinisation croisée a un impact plus accentuée sur le nombre de grains par épi chez $Z$. mays que l'autopollinisation, comme l'a mentionné Poehlman (1959). Les nombres moyens de grains par épi dans les lots 3 et 3' sont inférieurs à ceux des lots $1,2,4,1$ ', 2' et 4'. Malgré l'absence de la parthénocarpie chez le maïs, il y a eu quelques grains dans ces lots (3, 3'). Ceci pourrait s'expliquer par la détérioration du papier kraft au cours de la manipulation. En effet, durant l'investigation, les violentes pluies régulières ont crée de petits trous sur le papier kraft qui a probablement laissé passer quelques de pollen.

Tableau 5 : Rendement grainier de Zea mays dans les différents traitements à Maroua

\begin{tabular}{|c|c|c|c|c|c|c|c|c|c|c|}
\hline \multirow[t]{2}{*}{ Traitement } & \multirow[t]{2}{*}{ année } & \multicolumn{3}{|c|}{$N G / E$} & \multicolumn{3}{|c|}{$m G / E$} & \multicolumn{3}{|c|}{$N G T$} \\
\hline & & $n$ & $m$ & $S$ & $n$ & $m$ & $S$ & $N G A$ & $N G N$ & $P(\%)$ \\
\hline $\mathrm{L}_{1}$ & 2015 & 40 & 412,87 & 163,05 & 40 & 61,92 & 29,46 & 5842 & 10718 & 64,72 \\
\hline $\mathrm{L}_{2}$ & 2015 & 40 & 244,87 & 116,54 & 40 & 31,10 & 22,23 & 8435 & 1285 & 13,22 \\
\hline
\end{tabular}




\begin{tabular}{ccccccccccc}
\hline $\mathrm{L}_{3}$ & 2015 & 40 & 63,73 & 61,25 & 40 & 0,19 & 0,36 & 2549 & 0 & 00,00 \\
$\mathrm{~L}_{4}$ & 2015 & 40 & 265,57 & 134,07 & 40 & 34,31 & 24,11 & 1546 & 9094 & 85,47 \\
\hline $\mathrm{L}_{1}$, & 2016 & 40 & 418,56 & 60,95 & 40 & 62,47 & 27,56 & 5512 & 11248 & 67,27 \\
$\mathrm{~L}_{2}$, & 2016 & 40 & 230,25 & 67,84 & 30 & 32,13 & 19,47 & 7648 & 1592 & 17,23 \\
$\mathrm{~L}_{3}$, & 2016 & 40 & 71,18 & 38,56 & 40 & 0,17 & 0,13 & 2847 & 0 & 00,00 \\
$\mathrm{~L}_{4}$, & 2016 & 40 & 371,01 & 64,37 & 30 & 38,21 & 23,93 & 1910 & 12930 & 87,13 \\
\hline
\end{tabular}

$\boldsymbol{N G / E}:$ nombre de grains par épi ; $\boldsymbol{m} \boldsymbol{G} \boldsymbol{E}:$ masse de grains par épi ; $\boldsymbol{N G T}:$ nombre de grains total $; \boldsymbol{P}(\boldsymbol{\%})$ : pourcentage de grains normaux, $\mathbf{n}:$ nombre d'épi; NGA : nombre de grains avortés ; NGN : nombre de grains normauxm: moyenne; $s$ : écart-type $; \boldsymbol{L 1}, \mathbf{L 2}, \mathbf{L 3}$,

$\boldsymbol{L 4}, \boldsymbol{L 1}$ ', $\boldsymbol{L} \mathbf{2}^{\prime}, \boldsymbol{L} \mathbf{3}^{\prime}$ ' et $\boldsymbol{L} \mathbf{4}^{\prime}$ : lots 1, 2, 3, 4, 1', 2', 3' et 4' respectivement.

* Les différences entre les masses moyennes des grains par épi issues des quatre lots sont hautement significatives en $2015(F 2015$ $=58,30 ; d d l=3 ; P<0,001)$ et en $2016(F 2016=61,25 ; d d l=3$; $P<0,001)$. Les comparaisons entre les masses moyennes des grains par épi issus des lots 1 et $2(Z 2015=5,05 ; d d l=88 ; P<0,05)$ et des lots 1' et 2' $(Z 2016=8,05 ; d d l=88 ; P<0,05)$ révèlent des différences significatives. La contribution numérique de la masse moyenne des grains par épi attribuable aux insectes floricoles est de 49,17\%. En observant les masses moyennes des grains par épi des lots 4 et 4' et celles des lots 2 et 2', il apparait que les masses moyennes des grains par épi issue des lots 4 et 4' sont plus élevées que ceux des lots 2 et 2'. Ceci montre que la pollinisation croisée influence plus sur la masse des grains chez le maïs que l'autopollinisation, comme l'a signalé Poehlman (1959). Du tableau 5, il ressort que les masses moyennes de grains par épi dans les lots 3 et 3' sont largement inférieures à celles des lots 1,2, 4, 1', 2' et 4'. Cette masse moyenne des grains par épi insignifiante prouve que les quelques grains obtenus dans les lots 3 et 3 ' ne sont pas viable (Pitrat, 2015).

Les différences entre les pourcentages des grains normaux dans les quatre lots montrent une différence très hautement significative en $2015\left(\chi^{2} 2015=127,69 ; d d l=3 ; P<0,001\right)$ et en $2016\left(\chi_{2016}^{2}=127,69 ; d d l=3 ; P<0,001\right)$. Les différences sont hautement significatives entre les lots 1 et 2 d'une part $\left(\chi^{2} 2015=55,76\right.$; $d d l=1 ; P<0,005)$, puis entre les 1' et 2' d'autre part $\left(\chi^{2} 2016=87,34\right.$; $d d l=1 ; P<0,001)$. La contribution numérique dans le pourcentage des grains normaux dû à l'influence des insectes floricoles est de $76,48 \%$. Ce chiffre est largement supérieur à celui trouvé par Tchuenguem et al. (2009) qui est de $31,51 \%$ sur V. unguiculata. Ceci serait dû au fait que le niébé a un système de reproduction à prédominance autogame. Le taux de grains normaux est plus élevé 
dans les lots 4 et 4' que dans les lots 2 et 2' respectivement. Ceci s'explique par une prédominance de la pollinisation croisée sur l'autopollinisation, induisant de ce fait l'obtention de grains de bonne qualité chez le maïs (Marceau, 2010). L'absence de grain normal dans les lots 3 et 3 ' prouve ou confirme que la parthénocarpie n'existe par chez Z. mays.

\section{Conclusion}

Dix neuf espèces d'insectes ont visité les panicules de Z. mays pour récolter le pollen à Maroua. Parmi ces insectes, Amegilla sp. était de loin le plus prépondérant et le plus fréquent avec $95,30 \%$ des visites. Ces insectes butinaient les panicules de cette Poaceae dans la matinée entre $6 \mathrm{~h}$ et $11 \mathrm{~h}$, avec un pic d'activité situé entre $6 \mathrm{~h}$ et $7 \mathrm{~h}$, soit $89,44 \%$ des visites observées à cette tranche horaire. En comparant les rendements des plantes à inlorescences non protégées à ceux des plantes à panicules protégées des insectes, il est apparu que: (a) les insectes floricoles ont un impact positif sur le rendement en grains de $Z$. mays sur les plans quantitatif et qualitatif; (b) la pollinisation croisée influence positivement la quantité et la qualité des grains chez le maïs par rapport à l'autopollinisation. Le traitement des plants de maïs aux pesticides chimiques est à éviter pendant la période de floraison afin de bénéficier du service écosystémique des insectes pollinisateurs.

\section{Remerciements}

Les auteurs remercient: (a) l'Institut de Recherche Agronomique pour le Développement (IRAD) de Maroua pour avoir mis à notre disposition les Semences ; (b) M. Jacob Koue Kada et M. Justin Dessienbe pour leur aide physique lors de la préparation de la parcelle expérimentale.

\section{References:}

1. Arem, (2011). Enjeux de la pollinisation pour la production agricole en Tarn-Et-Garonne. INP. Toulouse. $106 \mathrm{p}$.

2. Borror D.J. et White RE., (1991). Les insectes de l'Amerique du Nord (au nord du Mexique). Les guides Peterson, (éd.), Broquet, $408 \mathrm{p}$.

3. Delvare G. et Arbelenc HP., (1989). Les insectes d'Afrique et d'Amérique tropicale. Clés pour la reconnaissance des familles. CIRAD (ed.), Montpellier, $297 \mathrm{p}$.

4. Dounia, Amada B., Douka C., Elono ASP., Ningatoloum C., Belinga BR., Gagni AF., Fomekong F., Tamesse JL. et Tchuenguem FF-N., (2018). Foraging Activity of Apis mellifera L. (Hymenoptera: Apidae) on Corn Panicles at Yaoundé, Cameroon. Canadian Journal of Agriculture and Crops, 3(2): 64- 71. 
5. DSCE, (2009). Document de stratégie pour la croissance et l'emploi. MINEPAT, Cameroun, $112 \mathrm{p}$.

6. Eardley CD., Kuhlman M. et Pauly A., (2010). Les genres et sous genres d'abeilles de l'Afrique sub saharienne. ABC Taxa 9, 152 p.

7. FAO, (2014). FAOSTAT data 2014 Data available at http://faostat3.fao.org; Agricultural data/Agricultural production/Crops primary.

8. Fichtl R. et Adi A., (1994). Honeybee flora of Ethiopia. Weikersheim, Germany: Margraf Verlag, 510 p.

9. Gallai N., Salles JM., Settele J. et Vaissière BE., (2009). Economic valuation of the vulnerability of world agriculture confronted with pollinator decline. Ecological Economics, 68 (3) : 810-821.

10. Hamel M-A. et Dorff E., (2014). Le maïs : troisième culture en importance au Canada. Statistique Canada, 17 p.

11. Klein AM., Vaissière BE., Cane JH., Steffan-Dewenter I., Counningham SA., Kremen C. et Tscharntke T., (2007). Importance of pollinators in changing landscapes for word crops. Proceeding of the Royal society, London (B), 274: 303-313.

12. Kremen C., Williams NM. et Thorp RW., (2002). Crop pollination from native bees at risk from agricultural intensification. Proceedings of the National Academy of Sciences of the USA, 99: 16812-16816.

13. Marceau A., (2010). Pollinisation inter-parcellaire chez le maïs: analyse et coupage des processus conditionnant la présence du pollen viable en fonction de la distance à la source. Thèse de doctorat. Institut des sciences et Industries du vivant et de l'environnement, Paris $143 \mathrm{p}$.

14. Michener CD., (1969). Notes on the nests and life histories of some African Halictid bees with description of a new species. Trans. Am. Entomol.Soc, 94: 473-497.

15. MINADER, (2012). Annuaire des Statistiques du Secteur Agricole, Campagnes 2009 \& 2010. Direction des Enquêtes et Statistiques, AGRI-STAT CAMEROUN n ${ }^{\circ} 17,123 \mathrm{p}$.

16. Pando JB., Tchuenguem FF-N. et Tamesse JL., (2011). Foraging and pollination behavior of Xylocopa calens Lapeletier (Hymenoptera: Apidae) onPhaseoluscoccineus L. (Fabaceae) flower at Yaoundé (Cameroon). Entomological Research. 41: 185-193.

17. Pauly A., (1984). Mission entomologique en Afrique occidentale (1979-80) : renseignements éco-biologiques concernant les Hyménoptères. Notes Faun. Gembloux11, 43 p.

18. Pauly A., (2014). Les Abeilles des Graminées ou LipotrichesGerstaecker, 1858, sensu stricto (Hymenoptera: Apoidea: 
Halictidae: Nomiinae) de l'Afrique subsaharienne.Belgian Journal of Entomology, 20: 1-393.

19. Pesson P. et Louveaux J., (1984). Pollinisation et production végétale. In cultures à graines oléagineuses des régions tempérées. INRA, Paris 363: 317-322.

20. Phaya T., (1985). Gathering corn pollen behaviour of honeybee (Apis mellifera L.)and pollen grain distribution. M.S. Thesis, Kasesot University of Bangkok, $144 \mathrm{p}$.

21. Pitrat M., (2015). La parthénocarpie: des fruits sans graines. Botanique-Jardins de France, 633 :1-2.

22. Poehlman J.M., (1959). Breeding field crops. New York: Holt, Rinehart \& Winston, USA, $427 \mathrm{p}$.

23. Schwartz D., (1984). Méthodes statistiques à l'usage des médecins et des biologistes. Flammarion Médecine-Science, 318 p.

24. Taséi JN., (1984). Cultures à graines oléagineuses des régions tempérées. In : Pollinisation et productions végétales (Pesson et Louveaux J., eds), Inra, Paris, 309-330 pp.

25. Tchuenguem FF-N. (1993). Activité des insectes anthophiles et son impact sur les rendements de deux plantes cultivées au Cameroun : Zea mays LINNE (Gramineae) etArachis hypogaea LINNE(Papilionaceae).Thèse, Départ. Biol. Physiol. Anim., Univ. Yaoundé 1,133 p.

26. Tchuenguem FF-N., Messi J. et Pauly A., (2001). Activité de Meliponula erythra sur les fleurs deDacryodes edulis et son impact sur la fructification. Fruits, $56: 179-188$.

27. Tchuenguem FF-N., Messi J. et Pauly A., (2002). L'activité de butinage des Apoïdes sauvages (Hymenoptera: Apoidea) sur les fleurs de maïs à Yaoundé (Cameroun) et réflexions sur la pollinisation des graminées tropicales. Biotechnologie, Agronomie, Société et Environnement, Gembloux, 6 (2) : 87 - 98.

28. Tchuenguem FF-N., Ngakou A. et Kegni BS., (2009). Pollination and yields responses of cowpea (Vigna unguiculata L. walp) to the foraging activity ofApis mellifera adansonii (Hymenoptera: Apidae) at Ngaoundéré (Cameroon). African Journal of Biotechnology, 8: 1988- 1996.

29. Vallardir F., (1964). Encyclopédie du monde végétal. Lidis (ed.), paris, Tome I, $568 \mathrm{p}$.

30. Zandonella P., (1984). Transport du pollen par les agents physiques : anémogamie et hydrogamie. In Pesson P., Louveaux J. (eds.). Pollinisation et productionsvégétales. INRA, Paris : 91-95. 